\title{
Enhanced Surveillance for National (Handicapped) Sports Games in Wakayama, Japan 2015
}

\section{Chisa Kambe1, Hiroko Fujii ${ }^{1}$, Tetsuya Niu1, Hideo Matsuura1, Naoko Nagai1, Yuuki Nakamura², Takako Nojiri ${ }^{3}$}

\author{
${ }^{1}$ Wakayama City Public Health Center, Wakayama, Japan \\ ${ }^{2}$ Graduate School of Pharmacy, Nihon University, Chiba, Japan \\ ${ }^{3}$ Wakayama Prefecture, Health Department, Wakayama, Japan \\ Email: contact@syndromic-surveillance.com
}

How to cite this paper: Kambe, C., Fujii, H., Niu, T., Matsuura, H., Nagai, N., Nakamura, Y. and Nojiri, T. (2018) Enhanced Surveillance for National (Handicapped) Sports Games in Wakayama, Japan 2015. Journal of Biosciences and Medicines, 6, 35-47.

https://doi.org/10.4236/jbm.2018.67004

Received: June 18, 2018

Accepted: July 21, 2018

Published: July 24, 2018

Copyright $\odot 2018$ by authors and Scientific Research Publishing Inc. This work is licensed under the Creative Commons Attribution International License (CC BY 4.0).

http://creativecommons.org/licenses/by/4.0/

(c) (i) Open Access

\begin{abstract}
Introduction: We undertook enhanced surveillance of the 2015 Kinokuni Wakayama National Sports Games $\left(70^{\text {th }}\right.$ National Sports Games) and the Kinokuni Wakayama Handicapped Sports Games $\left(15^{\text {th }}\right.$ National Handicapped Sports Games) held in Wakayama prefecture in 2015, for which this paper presented operational details and an evaluation. Methods: Enhanced surveillance conducted involved (Nursery) School Absenteeism Surveillance system ((N)SASSy), Prescription Surveillance, and Ambulance Transfer Surveillance from 30 August through 9 November. We evaluated those surveillances on all days including weekends, integrated the results as a daily report, and published it on the web. Results: We found no cluster or cases requiring more tracing and investigation except for suspected cases of rubella in (N)SASSy. Moreover, no moderate aberration was found for two consecutive days for the same area and symptom. Conclusion: Our experience with this enhanced surveillance presents important lessons for countermeasures by local governments for mass gatherings or politically important events.
\end{abstract}

\section{Keywords}

Enhanced Surveillance, National (Handicapped) Sports Games,

Mass-Gathering Event, (Nursery) School Absenteeism Surveillance System,

Prescription Surveillance, Ambulance Transfer Surveillance

\section{Introduction}

On the occasions of important international events, unusually large numbers of 
visitors enter the country and bioterrorism attacks become more probable. Therefore, earlier detection of outbreaks is quite important for mass gathering events such as the Olympic and Paralympic games, and FIFA World Cup tournaments, as well as politically high-profile events such as G7 summit meetings. Public health agents must conduct enhanced surveillance for such events [1]-[12].

In Japan, enhanced surveillance had been conducted in the past for the Kyushu Okinawa Group of Eight Summit (G8 summit) 2000 [4], the FIFA World Cup 2002 competition [5] [6], the 2008 Hokkaido Toyako G8 summit meeting [7], US President Obama's visit to Japan in 2009 [8], the Yokohama meeting of Asia-Pacific Economic Cooperation (APEC) in 2010, the Nagoya Tenth meeting of the Conference of the Parties (COP10) 2010 [9], the Fourth Japan-China-South Korea Trilateral Summit in 2011 [10], the Sports Festival in Tokyo in 2013 [11], the Uchinanchu festival in Okinawa 2016 [12], and the Ise-Shima G7 summit meeting of 2016. Particularly, enhanced surveillance for the Sports Festival in Tokyo in 2013 [11] was the first case of enhanced surveillance for national sports games. It was also important as preliminary practice for the Tokyo Olympic and Paralympic Games to be held in 2020.

Moreover, operations by local governments have been important for US President Obama's visit to Japan in 2009 [8], COP10 in 2010 [9], the Fourth Japan-China-South Korea Trilateral Summit in 2011 [10], and Uchinanchu festival in Okinawa in 2016 [12]. Such operations differ markedly from enhanced surveillance operations conducted by the central government for the G8 summit [4] [7] and the FIFA World Cup 2002 competition [5] [6].

This paper presents a description and an evaluation of enhanced surveillance conducted by local governments for the 2015 Kinokuni Wakayama national sports games ( $70^{\text {th }}$ National Sports Games) and the 2015 Kinokuni Wakayama Games ( $15^{\text {th }}$ National Handicapped Sports Games) during 26 September to 26 October, 2015 in Wakayama prefecture. Enhanced surveillance was conducted by interim surveillance and enforcements of the routine surveillance through reinforcement of the processes involved: sharing of information, daily assessment, daily situation reporting, and readiness for action when necessary. During the two Games, cumulative total of 0.76 million players, coaching staffs and audience visits were made, even though 0.95 million people reside in Wakayama prefecture.

We believe our experience of the enhanced surveillance as described below can provide a good example and important lesson for interim surveillance or countermeasure by local governments for mass gatherings or politically important events in Japan and in the rest of the world.

\section{Methods}

Enhanced surveillance was conducted using surveillance systems, (Nursery) School Absenteeism Surveillance system ((N)SASSy), Prescription Surveillance (PS), Ambulance Transfer Surveillance (ATS), and Official Syndromic Surveillance (OSS). All of them operate routinely except for ATS, in addition to 
case-based and sentinel surveillance operations covered (Official National Surveillance of Infectious Diseases (ONSID)) under the Infectious Diseases Control Law. This enhanced surveillance was operated by Wakayama prefecture and Wakayama city for analysis and evaluation with intensive information sharing among the related institutions in Wakayama prefecture.

\subsection{PS}

The system was developed through collaboration of the research group headed by Dr. Ohkusa, National Institute of Infectious Disease, and EM System Co. Ltd. [13]. It is currently operated by the Japan Medical Association, the Japan Pharmaceutical Association, School of Pharmacy, Nihon University, and EM System Co. Ltd.

Data for the number of prescriptions are extracted automatically and are analyzed daily through the data center of pharmacies, which monitors drugs categorized as "drugs for fever and pain relief", "drugs for common colds", "antibiotics", "anti-influenza virus drugs" (excluding amantadine), and "anti-Varicella-Zoster virus drugs", with the last two categories further sub-classified by patient age: "younger than 15", "16 - 64", and "older than 65 years old". As surveillance for potential bioterrorism using smallpox, we monitored unusual increasing in prescriptions of anti-Varicella-Zoster virus drugs to adults, without any increase in the population of children or elderly people.

As of the end of August, 2015, the number of pharmacies involved in PS were 23 in the Wakayama city public health center jurisdiction (PHCJ), 5 in the Iwade PHCJ, 3 in the Kainan PHCJ, 5 in the Hashimoto PHCJ, 8 in the Gobo PHCJ, 7 in the Shingu PHCJ, 8 in the Tanabe PHCJ, and 2 in the Yuasa PHCJ.

Aberrations in PS were defined at each participating pharmacy using historical analysis, by which the targeted drug prescription was regressed on the dummy variables for the epidemiological week number, the day of the week, holiday, the day after holidays, and a linear time trend. We recognized a higher aberration if today's number of prescriptions of the drug exceeded the predicted value from estimation using all data up to the prior days by five standard deviations of the prediction. Similarly, moderate aberration was found for four standard deviations; low aberration was found for three standard deviations. Aberrations at the community level were defined by integration of the aberrations at pharmacies in the community. We assigned a score of 1 to high aberration, 0.5 to moderate aberration, 0.25 to low aberration, and 0 to no aberration at each pharmacy. Subsequently, we calculated the average score over participating pharmacies in the community. For averages higher than 0.3 , we inferred high aberration in the community, inferring moderate for averages higher than 0.2 , and low for averages higher than 0.1 .

\section{2. (N)SASSy}

This unique surveillance is made available in Japan because the caregivers of 
students are required by schools to report the reasons for a child's absence from school activities. Caregivers are required by schools to report their child's symptoms before a diagnosis is made by a doctor. After being diagnosed by a doctor, they are required to report their diagnosis to schools as the reason for their child's absence. By reporting the certain type of diagnosed disease such as influenza or varicella from caregivers, the school can require that the student be "suspended" from school activities to prevent disease transmission inside a class, specific grade level, or school. Days during the suspended period are not counted against the total number of days required to advance to the next school grade. (N)SASSy is an internet-based system through which a school representative with access to the system makes a daily record of the reported information about infection-related absenteeism and suspensions. It was developed by the research group headed by Dr. Ohkusa, National Institute of Infectious Disease since 2007 [14] [15] and has keeping copyright, and currently operated by Japanese Society of School Health. It provides some general information (https://scl11.953862.net/schoolkoukai/view_all.php).

At that time, all municipal (nursery) schools and kodomoen, which are hybrid nursery school and kindergartens, in Wakayama city participated at (N)SASSy: 31 nursery schools, 13 kindergartens, 2 kodomoens, 56 elementary schools, and 19 junior high schools joined (N)SASSy. In Iwade city and Kinokawa city, all municipal elementary schools and junior high schools participated in (N)SASSy: 6 elementary schools and 2 junior high schools in Iwade city, and 6 elementary schools and 7 junior high schools in Kinokawa city joined.

We apply EARS [16] [17] to detect aberrations in the number of suspensions attributable to infection, and absences related to respective symptom in a city at three classifications. Low aberration was inferred as a day's number of patients was higher than the average of the prior seven days by three standard deviations: the original definition of $\mathrm{C} 1$ in EARS. Moderate aberration was inferred as four standard deviations higher. High aberration was inferred as five standard deviations higher.

\subsection{ATS}

ATS monitors the initial diagnosis when an ambulance arrives to deliver a patient to a hospital. In Japan, it was examined in an earlier study [18]; it has operated routinely in Tokyo [11]. However, in Wakayama prefecture, no routine ATS exists. Therefore, we created a temporary ATS using patient data in the widespread disaster and acute medicine information system in Wakayama prefecture, which uses initial diagnoses registered by ambulance crews. We defined the respective jurisdictions as $\mathrm{PHCJ}$.

We categorized initial diagnoses as fever, respiratory symptom, influenza and pneumonia, digestive symptoms, dizziness and impaired consciousness, convulsions, or bleeding. Aberration detection in ATS was similar to that with PS. The number of ambulance transferred by symptoms was regressed on the dummy 
variables for the epidemiological week number, the day of the week, holiday, the day after holidays, and a linear time trend. Then we inferred high-level aberration when the actual number of symptoms is higher than the fitted value by more than five times the standard deviation. Similarly, we inferred moderate level aberration it was when more than four times but fewer than five times. We also inferred low level aberration for more than three times but less than four times. Such a regression used data from January 1, 2014 to the present day because of data limitations.

\subsection{OSS}

OSS based on the Infectious Diseases Control Law started on April 1, 2008. When a physician at a designated medical facility (sentinel clinic or hospital) sees a patient meeting the reporting criteria, the facility must immediately make a report to the public health center via internet or fax. The report criteria are the following: 1) fever higher than $38^{\circ} \mathrm{C}$ and respiratory symptoms (except for external injury or organic cause) (respiratory symptoms refers to critical cases that must be admitted to a hospital), or 2) fever and rash or blistering skin disease (except for symptoms that are clearly attributable to diseases classified in categories II - IV, under the Law). Throughout the duration of the enhanced surveillance, fever and rash or blistering skin disease (the second criterion) were monitored especially closely for surveillance of bioterrorism using smallpox. Information in OSS was shared in the daily report during enhanced surveillance period.

\subsection{NOSSID}

NONSID, like OSS, is based on the Infectious Diseases Control Law. All, or sentinel medical institutions must report patients diagnosed by a doctor, and who are compatible with the reporting criteria. Information in NOSSID is shared in the daily report during enhanced surveillance period.

\subsection{Evaluation and Information Sharing}

We evaluated the surveillance on all days including weekends. We asked designated public health centers to collect more detailed information and responses if we found high aberration or moderate aberrations on two consecutive days in the same area for the same symptom or disease. The Wakayama City Public Health Center integrated the result as a daily report and shared information with the Health Promotion department in Wakayama prefecture and related facilities until noon. The information is also made available on the web at http://www.kansen-wakayama.jp/blog2/ and

http://www.pref.wakayama.lg.jp/prefg/041200/h_kansen/sabe.html/.

\subsection{Period}

The 2015 Kinokuni Wakayama National Sports Games were held from 26 September through 6 October, although swimming games were held 6 - 13 Septem- 
ber. The 2015 Kinokuni Wakayama Sports Games were held during 24 - 26 October. We conducted enhanced surveillance from 30 August through 9 November.

\subsection{Ex-Post Evaluation}

After enhanced surveillance ended, we interviewed the departments in Wakayama city related to National Sports Games for ex-post evaluation.

\subsection{Ethics}

No information obtained from enhanced surveillance was personally identifiable information such as names, addresses, or dates of birth. Moreover, we counted only patients with a certain condition from the collected information. Ethical guidelines for epidemiological research in Japan require no receipt of informed consent from patients under these circumstances.

\section{Results}

Table 1 presents the number of aberrations in PS in the upper panel and the number of aberrations in ATS in the lower panel. Table 2 shows the number of aberrations in symptoms of (N)SASSy in the upper panel and in diseases of (N)SASSy in the lower panel.

Table 1. Number of aberrations in PS and ATS.

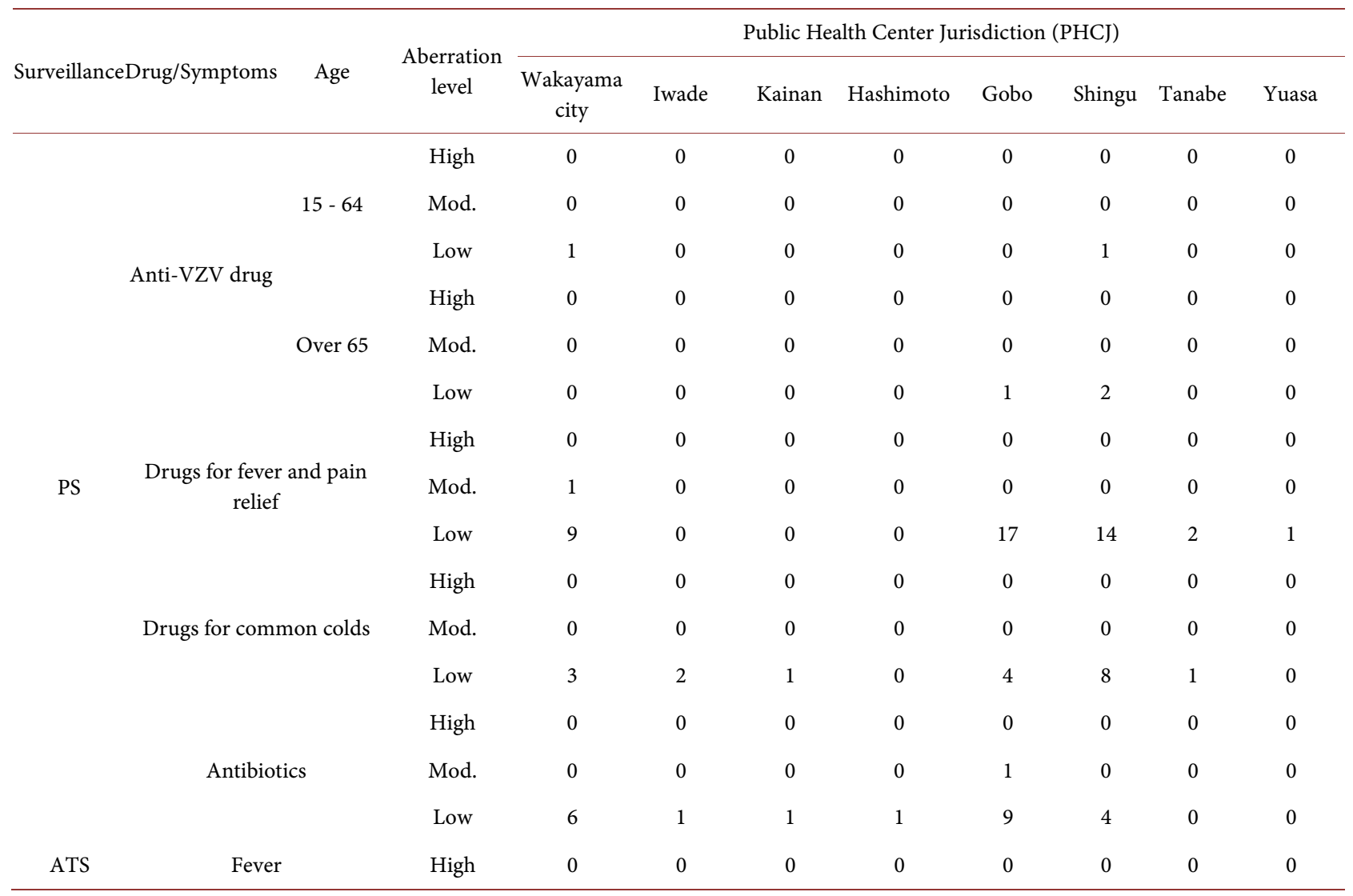




\section{Continued}

\begin{tabular}{ccccccccccc} 
& Mod. & 0 & 1 & 0 & 0 & 0 & 0 & 0 & 0 \\
& Low & 0 & 0 & 4 & 0 & 0 & 0 & 0 & 0 \\
\multirow{3}{*}{ Respiratory symptoms } & High & 0 & 0 & 0 & 0 & 0 & 0 & 0 & 0 \\
\multirow{3}{*}{ Bleeding } & Mod. & 0 & 0 & 0 & 0 & 0 & 0 & 0 & 0 \\
& Low & 1 & 0 & 0 & 0 & 0 & 0 & 0 & 0 \\
& High & 0 & 0 & 0 & 0 & 0 & 0 & 0 & 0 \\
& Mod. & 0 & 0 & 0 & 0 & 0 & 0 & 0 & 0 \\
& Low & 0 & 0 & 1 & 0 & 0 & 0 & 0 & 0 \\
\hline
\end{tabular}

Note: Mod., moderate aberration level; VZV, Varicella-Zoster virus; PS, prescription surveillance; ATS, ambulance transfer surveillance. We omitted lines from the table if no aberration occurred in the public health center jurisdiction. Aberrations in PS were defined at each participating pharmacy using historical analysis by which the targeted drug prescription was regressed on the dummy variables for the epidemiological week number, day of the week, holiday, day after holidays, and a linear time trend. We recognized a higher aberration if today's number of prescriptions of the drug exceeded the prediction from the estimation using all data up to the prior day by five standard deviations of the prediction. Similarly, moderate aberration was found for four standard deviations; low aberration was found for three standard deviations. Aberrations at the community level were defined by integration of the aberrations at pharmacies in the community. We assigned scores to high aberration as 1 , to moderate aberration as 0.5 , to low aberration as 0.25 , and to no aberration as 0 . Then we calculated the average score over participating pharmacies in the community. If the average was higher than 0.3 , then we defined high aberration in the community, moderate as 0.2 , and low as 0.1 . Similarly, aberrations in ATS were defined by which the number of ambulance transferred by symptoms was regressed on the dummy variables for the epidemiological week number, the day of the week, holiday, the day after holidays, and a linear time trend. We inferred high-level aberration as an actual number of symptoms higher than the fitted value by more than five times of standard deviation. Similarly we inferred moderate-level aberration for more than four times but fewer than five times. We also inferred low-level aberration when more than three times but less than four times. Such a regression used data from January 1, 2014 to the present day.

Table 2. Number of aberration in (N)SASSy aberration.

\begin{tabular}{|c|c|c|c|c|c|}
\hline & Symptom/Disease & $\begin{array}{c}\text { Aberration } \\
\text { Level }\end{array}$ & Wakayama city & Iwade city & Kinokawa city \\
\hline & & High & 0 & 2 & 0 \\
\hline & Fever & Moderate & 0 & 6 & 0 \\
\hline & & Low & 1 & 9 & 3 \\
\hline & & High & 0 & 0 & 0 \\
\hline & Headache & Moderate & 0 & 0 & 0 \\
\hline & & Low & 0 & 1 & 0 \\
\hline & & High & 0 & 0 & 0 \\
\hline \multirow[t]{9}{*}{ Symptoms } & Respiratory Symptom & Moderate & 0 & 1 & 0 \\
\hline & & Low & 0 & 2 & 0 \\
\hline & & High & 0 & 0 & 0 \\
\hline & Diarrhea & Moderate & 0 & 0 & 0 \\
\hline & & Low & 0 & 2 & 0 \\
\hline & & High & 0 & 0 & 0 \\
\hline & Others & Moderate & 0 & 0 & 0 \\
\hline & & Low & 0 & 3 & 0 \\
\hline & & High & 0 & 0 & 1 \\
\hline \multirow[t]{2}{*}{ Diseases } & Influenza & Moderate & 0 & 0 & 0 \\
\hline & & Low & 0 & 0 & 0 \\
\hline
\end{tabular}




\section{Continued}

\begin{tabular}{|c|c|c|c|c|}
\hline \multirow{3}{*}{ Varicella } & High & 1 & 0 & 0 \\
\hline & Moderate & 1 & 0 & 0 \\
\hline & Low & 2 & 0 & 0 \\
\hline \multirow{3}{*}{ Mumps } & High & 0 & 0 & 0 \\
\hline & Moderate & 1 & 0 & 0 \\
\hline & Low & 0 & 0 & 0 \\
\hline \multirow{3}{*}{$\begin{array}{c}\text { Epidemic } \\
\text { kerato-conjunctivitis }\end{array}$} & High & 0 & 0 & 0 \\
\hline & Moderate & 2 & 0 & 0 \\
\hline & Low & 0 & 0 & 0 \\
\hline \multirow{3}{*}{ Infectious gastroenteritis } & High & 0 & 0 & 0 \\
\hline & Moderate & 1 & 0 & 0 \\
\hline & Low & 0 & 0 & 0 \\
\hline \multirow{3}{*}{$\begin{array}{l}\text { Hemolytic streptococcal } \\
\text { infection }\end{array}$} & High & 0 & 0 & 0 \\
\hline & Moderate & 0 & 0 & 0 \\
\hline & Low & 1 & 0 & 0 \\
\hline \multirow{3}{*}{ HFMD } & High & 0 & 0 & 0 \\
\hline & Moderate & 0 & 0 & 0 \\
\hline & Low & 1 & 0 & 0 \\
\hline \multirow{3}{*}{ Others } & High & 1 & 0 & 0 \\
\hline & Moderate & 0 & 0 & 0 \\
\hline & Low & 0 & 0 & 0 \\
\hline
\end{tabular}

Note: HFMD denotes hand-foot-mouth disease. We omitted the lines from the table if no aberration was found for any public health center jurisdiction. Low aberration was defined as a day's number of patients being higher than the average of the prior seven days by three standard deviations. Moderate aberration was defined as four standard deviations higher. High aberration was defined as five standard deviations higher

\subsection{PS}

There was no high aberration. Moderate aberrations were found in drugs for fever and pain relief, and antibiotics.

\section{2. (N)SASSy}

High aberration in diseases was found for influenza, varicella and others, which was a suspected case of rubella. Moderate aberrations were found in varicella, mumps, two cases of epidemic kerato-conjunctivitis, and infectious gastroenteritis. Regarding symptoms, high aberration in fever was found two times. Moderate aberration was found in fever six times, and in respiratory symptoms.

During the period, a prefectural junior high school closed a class because of pharyngoconjunctival fever. However, this school did not participate in (N)SASSy at that time because it was a prefectural school, unmonitored by (N)SASSy. No other class closure occurred. The information of the suspected rubella case was shared among concerned participants. 


\subsection{ATS}

No high aberration was found. One moderate aberration was observed for fever. Four low aberrations were found in fever and one in respiratory symptoms, with one related to bleeding.

\subsection{OSS and NOSSID}

There was no report to OSS. NOSSID provided information from sentinels to daily reports on Wednesday.

\subsection{Evaluation and Information Sharing}

During the period, until 9 a.m. all information in the enhanced surveillance was collected, analyzed and evaluated every day including weekends. Daily reports were sent to concerned participants by 9:30 a.m. and were uploaded on the web site.

When we recognized high aberration, we did information collection. However, we found no cluster or case that needed more tracing and investigation except for the suspected rubella case in (N)SASSy. Moreover, no moderate aberration was found in two consecutive days for the same area and symptom.

\subsection{Ex-Post Evaluation}

We received the following advice and evaluation from some departments related to National Sports Games in Wakayama city.

1) We were able to elucidate the situation in Wakayama city using information from (nursery) schools, pharmacies, and ambulance transfers. Moreover, we were able to recognize the situation in areas surrounding Wakayama city. We were able to operate Kinokuni Wakayama National Sports Games with a safe conscience (National Sports Games, General Affairs Division).

2) No outbreak occurred. Events for which early response was necessarily were those in the nursery school and kodemoen. It was good chance to conventionalize data entry within a day and to check the area situation (Nursery and Kodomoen Division).

3) We usually learn about situations from the mass media. Therefore, daily reports of enhanced surveillance are expected to be helpful for the event organization division (Secretary Division).

4) It was useful to know about situations earlier than official announcements of ONSID (Fire Defense Division).

5) It was useful for recognition of area situations (Management for Health and School-provided Lunch Division).

6) Information related to ambulance transfers, infectious diseases, and school absenteeism was grasped by the respective divisions. Information integration through enhanced surveillance for this National Sports Games can be visualized comprehensively. Moreover, divisions that are uninterested in the monitoring of infectious diseases nevertheless acknowledged the role of surveillance for infec- 
tious diseases (General Affairs and Planning Division).

\section{Discussion}

\subsection{Period}

In general, enhanced surveillance was conducted from two weeks before event opening until two weeks after event closing because the incubation period of small pox, chicken pox or measles were estimated approximately $10-14$ days [11] [12]. Therefore, our starting date of enhanced surveillance on 30 August was about one week before the opening of swimming games, which was somewhat late. We had to start it on the 23 August. Conversely, it ceased in the two weeks after the 2015 Kinokuni Wakayama National Handicapped Sports Games closed, appropriately.

\subsection{PS}

Because there were 450 pharmacies in Wakayama prefecture as of 2012, the PS coverage rate was $14 \%$, which is lower than national average. Even though we did not have high aberrations that required investigation, we did not construct a framework for investigation among pharmacies or medical institutions. Such a framework remains as a future challenge.

PS is unique in the sense of providing nationwide covering. Moreover, our experience demonstrated that it would be useful as enhanced surveillance by local government for mass gatherings such as National Sports Games if the remaining challenges could be solved.

\section{3. (N)SASSy}

During 11 weeks, 3 of 5 high aberrations and 7 of 12 moderate aberrations occurred on Monday or the day of open consecutive holidays. This phenomenon probably reflects medical care-seeking patterns of patients: They wait patiently or suffer through a weekend or holiday and then rush to medical institutions on Monday or the day of open consecutive holidays. This phenomenon was also confirmed by PS [11] [13]. We must be reminded of this tendency when evaluating some aberration from (N)SASSy.

During the period, a prefectural junior high school closed a class because of pharyngoconjunctival fever. However, this school was not participating in (N)SASSy at that time because it was a prefectural school. Although we collected information from the school directly, if it had participated in (N)SASSy, we could have recognized the outbreak earlier and could have applied a response quickly before class closing became necessary [19]. In fact, (N)SASSy mainly covers city-managed public schools. Therefore, it is blind to events that occur at prefectural or private schools. Outbreaks sometimes occur in some schools that are not covered by (N)SASSy. There, earlier detection and timely response are difficult [19].

Schools in only three cities and nursery schools, kindergartens and kodo- 
moens in Wakayama city participated in (N)SASSy during the period. Subsequently, all schools in Wakayama prefecture joined (N)SASSy in 2017. However, nursery schools, kindergarten and kodomoens in areas other than Wakayama city are not covered by (N)SASSy. To prevent outbreaks at nursery schools, kindergarten and kodomoens, their participation remains as a future challenge.

For the suspected rubella case, we performed a tracing investigation. The epidemiological investigation and test results revealed that it was negative. We recognized through this experience that (N)SASSy is a powerful tool for encouraging information sharing and timely responses including tracing and investigation. Nevertheless, rubella is a notifiable disease in NOSSID. Multiple routes of recognition, NOSSID and (N)SASSy, are valuable for public health to avoid delay or halting of information.

Although (N)SASSy is a powerful method to avoid outbreaks in (nursery) schools and communities, our experience revealed that it is useful as enhanced surveillance operated by local government for mass gathering events including National Sports Games.

\subsection{ATS}

ATS was not operated routinely in Wakayama prefecture. It was constructed specifically for enhanced surveillance using information in the widespread disaster and acute medicine information system in Wakayama prefecture. Routine ATS has been operated only in Tokyo [11]; ATS for COP10 [9] or Toyako Summit [7] were also conducted temporarily. We asked ambulance crews to enter the patients' symptoms into the system in the two temporarily ATS, which might have imposed some burden on them. By contrast, in Wakayama prefecture, they routinely enter the patients' symptoms into the widespread disaster and acute medicine information system in Wakayama prefecture. Therefore, ATS in this enhanced surveillance did not impose an extra burden on ambulance crews. Although all prefectures have the widespread disaster and acute medicine information systems, their contents are not the same. For that reason, we do not know whether it includes patients' symptoms as Wakayama prefecture does. For example, when the enhanced surveillance was conducted for Uchinachu festival in Okinawa, Okinawa prefecture could not perform it [12] because the widespread disaster and acute medicine information systems in Okinawa prefecture did not include patient symptoms information. This experience in ATS is expected to provide a good example when prefectures other than Tokyo introduce ATS. We are seeking to construct routine ATS in areas other than Tokyo. The system is also expected to contribute to enhanced surveillance for events including the National Sports Games.

Some aberrations occurred when the number of transfers was only one case in an area in a single day. The reason might be that we were unable to conduct basic research using data in Wakayama prefecture because of time limitations. We had to adjust the definition of aberration targeted symptoms and areas, and confirm them to avoid such cases. 


\subsection{Evaluation and Information Sharing}

Although we were able to operate enhanced surveillance, evaluate them, make daily report and upload it without no trouble, some meetings must be held with concerned parties during the period to exchange opinions and to adjust the enhanced surveillance if necessary.

As symbolized by the opinion "We were able to operate Kinokuni Wakayama National Sports Games with a safe conscience", the most important contribution of our enhanced surveillance was the safe conscience of all concerned parties, local residents, and players at these popular events.

\section{Conclusion}

We administered enhanced surveillance for the 2015 Kinokuni Wakayama National Sports Games and the 2015 Kinokuni Wakayama National Handicapped Sports Games using ATS using the widespread disaster and acute medicine information system in Wakayama prefecture, and PS and (N)SASSy as well as OSS, which had been used for the Sports Festival Tokyo in 2013. Particularly, no routine ATS is used in areas other than Tokyo. Our experience is expected to be a good example for use when prefectures other than Tokyo introduce ATS. We hope that it will also contribute to enhanced surveillance by local governments for events including National Sports Games. Our experience with this enhanced surveillance is expected to be an important lesson for countermeasure by local governments for mass gatherings or politically important events in the rest of the world as well as the World Cup in Rugby Football of 2019 or the Tokyo Olympic Paralympic Games of 2020 in Japan.

\section{Acknowledgements}

We acknowledge all participants of the enhanced surveillance including (nursery) schools, pharmacies, and ambulance crews.

\section{Conflict of Interest}

No author has any conflict of interest, financial or otherwise, related to this study.

\section{References}

[1] WHO (2015) Public Health for Mass Gatherings: Key Considerations.

[2] Dafni, U.G., Tsiodras, S., et al. (2004) Algorithm for Statistical Detection of Peaks-Syndromic Surveillance System for the Athens 2004 Olympic Games. Morbidity and Mortality Weekly Report, 53, 86-94.

[3] Jorm, L.R., Thackway, S.V., Churches, T.R., et al. (2003) Watching the Games: Public Health Surveillance for the Sydney 2000 Olympic Games. Journal of Epidemiology and Community Health, 57, 102-108. https://doi.org/10.1136/jech.57.2.102

[4] Osaka, K., Takahashi, H. and Ohyama, T. (2002) Testing a Symptom-Based Surveillance System at High-Profile Gatherings as a Preparatory Measure for Bioterrorism. Epidemiology and Infection, 129, 429-434. 
https://doi.org/10.1017/S0950268802007689

[5] Suzuki, S., Ohyama, T., Taniguchi, K., et al. (2003) Syndromic Surveillance of Infectious Diseases during the World Cup Football Games Held in Korea and Japan, May-July 2002. Infectious Agents Surveillance Report, 24, 37-38. (In Japanese)

[6] Kamiya, N., Ikeda, K., Nadaoka Y., et al. (2002) The Syndrome Surveillance during FIFA 2002 World Cup TM. Annual Report of the Tokyo Metropolitan Research Laboratory of Public Health, 53, 287-292. (In Japanese)

[7] Ohkusa, Y., Yamaguchi, R., Sugiura, H., et al. (2009) 2008 G8 Hokkaido Toyako Summit Meeting Syndrome Surveillance. Kansenshogaku Zasshi, 83, 236-244. (In Japanese) https://doi.org/10.11150/kansenshogakuzasshi.83.236

[8] Ohkusa, Y., Sugawara, T., Masuda, K., et al. (2010) Enhanced Surveillance for US Presidential Visit to Japan. Kansenshogaku Zasshi, 84, 708-713. (In Japanese) https://doi.org/10.11150/kansenshogakuzasshi.84.708

[9] Inaba, S., Ohkusa, Y., Sugawara, T., et al. (2012) Operation and Evaluation of Enhanced Surveillance for COP10 at Nagoya in 2010. Japanese Journal of Disaster Medicine, 17, 326-333. (In Japanese)

[10] Sugishita, Y., Ohkusa, Y., Sugawara, T., et al. (2013) Enhanced Syndromic Surveillance for the Fourth Japan-China-South Korea Trilateral Summit 2011. Journal of Bioterrorism and Biodefense, 4, 126. https://doi.org/10.4172/2157-2526.1000126

[11] Shimatani, N., Sugishita, Y., Sugawara, T., et al. (2015) Enhanced Surveillance for Sports Festival in Tokyo 2013: Preparation for Tokyo 2020 Olympic and Paralympic Games. Japanese Journal of Infectious Diseases, 68, 288-295. https://doi.org/10.7883/yoken.JJID.2014.233

[12] Yamakawa, M., Yamauchi, M., Nidaira, M., et al. (2017) Enhanced Public Health Surveillance for the Sixth Worldwide Uchinanchu Festival Conducted by the Okinawa Prefectural Government. Japan Journal of Biosciences and Medicines, 5, No. 9.

[13] Ohkusa, Y., Sugawara, T., Taniguchi, K., et al. (2011) Real-Time Estimation and Prediction for Pandemic A/H1N1 (2009) in Japan. Journal of Infection and Chemotherapy, 17, 468-472.

[14] Sugiura, H., Hata, T., Kodama, K., Oikawa, K., Imamura, T., Ohkusa, Y. and Okabe, N. (2010) Effectiveness of Class Closure for Influenza Pandemic in 2009 Using Schools Absenteeism Surveillance System. Gakkouhokenkenkyu, 52, 214-218. (In Japanese)

[15] Ohkusa, Y., Sugawara, T., Mitani, M., Sugiura, H. and Okabe, N. (2011) Construction of Schools Absenteeism Surveillance System and Evaluation. Gakkouhokenkenkyu, 53, 312-319. (In Japanese)

[16] Hultiwagner, L., Thompson, W., Seeman, G.M., et al. (2003) The Bioterrorism Preparedness and Response Early Aberration Reporting System (EARS). Journal of Urban Health, 80, 89-96.

[17] Hultiwagner, L., Browne, T., Seeman, G.M., et al. (2005) Comparing Aberration Detection Methods with Simulated Data. Emerging Infectious Diseases, 11, 314-316. https://doi.org/10.3201/eid1102.040587

[18] Ohkusa, Y., Kawaguchi, Y., Sugawara, T., et al. (2006) Basic Research for Syndromic Surveillance Using Ambulance Transfer Data. Journal of Japan Association of Acute Medicine, 17, 712-720. (In Japanese)

[19] Kurita, J., Sugawara, T., Matsumoto, K., Nakamura, Y. and Ohkusa, Y. (2018) Association among (Nursery) School Absenteeism Surveillance System and Incidence of Infectious Diseases. School Health, 14, 21-27. 